\title{
Increased expression of osteopontin gene in atypical teratoid/rhabdoid tumor of the central nervous system
}

\author{
Chung-Lan Kao ${ }^{1,7}$, Shih-Hwa Chiou ${ }^{2,8,9}$, Yann-Jang Chen ${ }^{3}$, Sher Singh ${ }^{4}$, Han-Tso Lin ${ }^{2,5}$, \\ Ren-Shyan Liu ${ }^{5}$, Chih-Wen Lo ${ }^{6}$, Chi-Chang Yang ${ }^{6}$, Chin-Wen Chi ${ }^{2}$, Chen-hsen Lee ${ }^{2}$ \\ and Tai-Tong Wong ${ }^{6}$
}

\footnotetext{
${ }^{1}$ Department of Physical Medicine \& Rehabilitation; ${ }^{2}$ Department of Education and Medical Research, Taipei Veterans General Hospital, Taiwan, ROC; ${ }^{3}$ Department of Life Science, National Yang-Ming University, Taiwan, ROC; ${ }^{4}$ National Science and Technology, Center for Disaster Reduction, Taiwan, ROC; ${ }^{5}$ Department of Nuclear Medicine, Taipei Veterans General Hospital, Taiwan, ROC; ${ }^{6}$ Division of Pediatric Neurosurgery, The Neurological Institute, Taiwan, ROC; ${ }^{7}$ Institute of Clinical Medicine; ${ }^{8}$ Department of Opthalmology, National Yang-Ming University, Taiwan, ROC and ${ }^{9}$ National Research Institute of Chinese Medicine, Taiwan, ROC
}

\begin{abstract}
The atypical teratoid/rhabdoid tumor, primary to the central nervous system, is a highly malignant and aggressive neoplasm of infancy and childhood. Although having distinct biological features and clinical outcomes, it is frequently misdiagnosed as primitive neuroectodermal tumor/medulloblastoma. To further distinguish the underlying pathogenesis and to identify biological markers for clinical use, an atypical teratoid/ rhabdoid tumor-derived cell line was established and its gene expression pattern analyzed in comparison to the human astrocyte SVG12 cell line and the human DAOY medulloblastoma cell line using a complementary DNA microarray method. The osteopontin gene was found specifically upregulated in atypical teratoid/rhabdoid tumor cells. This specificity was confirmed by immunohistochemistry in pathological sections of tissues from atypical teratoid/rhabdoid tumor patients. Even though the role of osteopontin in the cytopathogenesis of atypical teratoid/rhabdoid tumor still needs to be determined, our data support that overexpressed osteopontin is a potential diagnostic marker for atypical teratoid/rhabdoid tumor.
\end{abstract}

Modern Pathology (2005) 18, 769-778, advance online publication, 18 March 2005; doi:10.1038/modpathol.3800270

Keywords: atypical teratoid/rhabdoid tumor; medulloblastoma; microarray; osteopontin; real-time RT-PCR

The atypical teratoid/rhabdoid tumor (AT/RT), primary to the central nervous system, is a highly malignant tumor occurring in patients most commonly under 3 years of age, and often fatal within 1 year after diagnosis. ${ }^{1-3}$ Histologically, AT/RT consists of a unique combination of rhabdoid cells as well as neuroepithelial, peripheral epithelial and mesenchymal elements. ${ }^{1-3}$ In the past, the majority of AT/RT was misclassified as primitive neuroectodermal tumor/medulloblastoma at supratentorial sites because of similarities in radiological and histological features of these two tumors. ${ }^{1,2}$ The

Correspondence: Dr S-H Chiou, MD, PhD, Department of Education and Medical Research, Taipei Veterans General Hospital, Taiwan, ROC.

E-mail: shchiou@vghtpe.gov.tw

Received 23 March 2004; revised 6 July 2004; accepted 8 July 2004; published online 18 March 2005 immunophenotypic diversity of AT/RT could also be easily mistaken for germ cell tumors. ${ }^{4}$

Although controversial, cytogenetic study is currently used to differentiate between brain tumors (including AT/RTs). Previous studies have demonstrated $17 \mathrm{p}$ loss in $25-50 \%$ medulloblastomas, but not in AT/RT. ${ }^{5,6}$ Rorke et al further found that the chromosomal abnormality identified in a subset of AT/RT is monosomy or contains a deletion of chromosome $22 .{ }^{7}$ Recent studies reported that a CNS rhaboid tumor with an unbalanced 9;22 translocation leads to loss of 22q11. ${ }^{8}$ Subsequently, hSNF5/INI1 gene was identified on $22 \mathrm{q} 11.2$ as a potential tumor suppressor gene responsible for the oncogenesis of AT/RT. ${ }^{9}$ In addition, some studies also detect rearrangement of chromosomes 6 and 11 and a reciprocal translocations on $(12 ; 22)(q 24.3 ; q 11.2-12) .{ }^{10,11}$ Thus, there remains inconsistencies in AT/RT-associated somatically 
acquired chromosome abnormalities and questions regarding the histogenesis and biological determinants of malignancy in AT/RT.

Because AT/RT is still a rather unfamiliar pathological entity, investigation of its underlying pathogenesis and identification of potential markers for diagnosis are urgently needed. In this study, microarray assays and real-time RT-PCR were used to clarify the distinct gene expression profile of an AT/ RT-derived cell line. We found that the expression of the osteopontin $(O P N)$ gene was specifically upregulated in AT/RT cells. The increased protein expression in the clinical specimens of AT/RT was confirmed by immunohistochemistry. These findings provide valuable information about $O P N$ as a potential diagnostic marker for AT/RT.

\section{Materials and methods}

\section{Tumor Cell Culture}

This research follows the tenets of the Declaration of Helsinki and has been reviewed by Institutional Review Committee at Taipei Veterans General Hospital. All samples were obtained after patients gave their informed consent. The human astrocyte cell line (SVG12) and DAOY metastatic medulloblastoma cell line (HTB-186) were obtained from American Type Culture Collection (ATCC Manassas, VA, USA). The AT/RT tissue was dissected into $2-3 \mathrm{~mm}$ segments and digested by collagenase A (Liberase, Roche, Indianapolis, IN, USA). The suspended cells were washed with phosphatebuffered saline (PBS, $\mathrm{pH}$ 7.2) and treated with $0.025 \%$ trypsin-EDTA (GIBCO, Grand Island, NY, USA) in Hank's balanced salt solution (Sigma, St Louis, MO, USA) for $15 \mathrm{~min}$ at $37^{\circ} \mathrm{C}$, and then passed through a $30-\mu \mathrm{m}$ mesh nylon screen. The filtrate was centrifuged $(800 \mathrm{~g}, 5 \mathrm{~min})$ and the resulting cell pellet was resuspended and seeded into a T75 flask (Corning, Corning, NY, USA). Cultures were grown in DMEM (Biosource, Camarillo, CA, USA) containing 10\% heat-inactivated fetal bovine serum, $2 \mathrm{mM}$ glutamine, penicillin (100 U/ $\mathrm{ml})$ and streptomycin $(100 \mu \mathrm{g} / \mathrm{ml})$. The cells were incubated at $37^{\circ} \mathrm{C}$ in an atmosphere of $5 \% \mathrm{CO}_{2}$. Within 5 days, cultures were nearly confluent and were passaged 1:4.

\section{Comparative Genomic Hybridization (CGH)}

The CGH procedure was as previously described..$^{12}$ Metaphase spreads from normal human lymphocytes were prepared using standard protocols. Briefly, the slides were aged for 2-3 days before denaturation at $72^{\circ} \mathrm{C}$ in $70 \%$ spectrum red formamide $/ 2 \times$ saline sodium citrate (SSC), followed by dehydration in a graded series of ethanol. The slides were treated with proteinase K (Sigma, USA) at a concentration of $0.1 \mu \mathrm{g} / \mathrm{ml}$ in $20 \mathrm{mM}$ Tris ( $\mathrm{pH} 7.5$ )/
$2 \mathrm{mM} \mathrm{CaCl}{ }_{2}$ before hybridization. Nick-translated, spectrum red-labeled tumor DNA and spectrum green-labeled normal DNA were co-precipitated with excess unlabeled human Cot-1 DNA (GibcoBRL, Grand Island, NY, USA), denatured, and hybridized to the normal metaphase slide preparations. In all, 10-12 images were captured and analyzed by a Cytovision workstation. The threshold indicated that gain and loss were to be set at 1.2 and 0.8 , respectively.

\section{Microarray Gene Expression Analysis}

The total RNA was extracted from AT/RT, astrocyte SVG-12, and Daoy medulloblastoma cells using Trizol reagent (Life Technologies, Bethesda, MD, USA) and the Qiagen Rneasy (Qiagen, Valencia, CA, USA) column for purification. The total RNA was reverse-transcribed with Superscript II Rnase H-reverse transcriptase (Gibco BRL) to generate Cy3and Cy5-labeled (Amersham Biosciences Co., Piscataway, NJ, USA) cDNA probes for control and treated samples, respectively. The labeled probes were hybridized to a cDNA microarray containing 7500 immobilized cDNA fragments. Fluorescence intensities of Cy3 and Cy5 targets were measured and scanned separately using GenePix 4000B Array Scanner (Axon Instruments, Burlingame, CA, USA). Data analysis was performed using GenePix Pro 3.0.5.56 (Axon Instruments, USA) and GeneSpring V5.1 (Silicon Genetics Corp., Redwood City, CA, USA). Microarray data normalization was performed as described. ${ }^{13}$ The results were normalized for the labeling and detection efficiencies of the two fluorescent dyes, then used to determine differential gene expression between AT/RT and astrocyte SVG-12, and between AT/RT and Daoy medulloblastoma cells.

\section{Real-Time RT-PCR}

Relative quantitation by real-time RT-PCR was performed using SYBR-green detection of PCR products in real time using the LightCycler (Roche Molecular Systems, Alameda, CA, USA). Quantification in the unknown samples was performed by the LightCycler Relative Quantification Software version 3.3 (Roche Molecular Systems). Briefly, total RNA $(1 \mu \mathrm{g})$ of each sample was reverse transcribed in $20 \mu \mathrm{l}$ using $0.5 \mu \mathrm{g}$ of oligo dT and $200 \mathrm{U}$ Superscript II RT (Invitrogen, Carlsbad, CA, USA). Amplification was carried out in a total volume of $20 \mu \mathrm{l}$ containing $0.5 \mu \mathrm{M}$ of each primer, $4 \mathrm{mM} \mathrm{MgCl}_{2}$, $2 \mu \mathrm{l}$ LightCycler ${ }^{\mathrm{TM}}$-FastStart DNA Master SYBR green I (Roche Diagnostics $\mathrm{GmbH}$, Mannheim, Germany) and $2 \mu \mathrm{l}$ of 1:10 diluted cDNA. In each experiment, the human $\beta 2$-microglobulin (B2M) housekeeping gene was amplified as a reference standard. B2M primers were as follows: B2Mf, 5'-CTCGCGCTACTCTCTCTTTCTG-3' (nt 41-62, 
GenBank Accession no. NM 004048), and B2Mr, 5'GCTTACATGTCTCGATCCCACTT-3' (nt 375-353). Other target gene primers were as follows: Osteopontin(f), 5'-TGAGAGC AATGAGCATTCCGATG-3' (nt 822-844, GenBank Accession no. J04765), Osteopontin(r), 5'-CAGGGAGTTTCCATGA AGCCAC-3' (nt 1196-1175). MMP2(f), 5'-GATCTTCTTCTT CAAGGACCGG-3' (nt 1741-1762, GenBank Accession no. NM_004530), MMP2(r), 5'-TTGGGAAA GCCAGGATCCAT-3' (nt 2100-2081). Decorin(f), $5^{\prime}$-AGCTGAAGGAATTGCCAGAA-3' (nt 460-479, GenBank Accession no. BC005322), Decorin(r), 5'-TGGTGCCCAGTTCTATGACA-3' (nt 591-572). Lumican(f), $5^{\prime}$-CCACAACAACCTGACAGAGT-3' (nt 513-532, GenBank Accession no. NM_002345), Lumican(r), 5'-CAAGTTGATTGACCTCCAGG-3' (nt 1000-981). PDGFRA(f), 5'-GTCTACGAGATCATGG TGAAATGC-3' (nt 3167-3190, GenBank Accession no. NM 006206 ), PDGFRA(r), 5'-AATGGCACTCT CTTCAGAGGTCT G-3' (nt 3546-3521). Calponin 1(CNN1)(f), 5'-CATGACTGCC TACGGCACGA-3' (nt 786-805, GenBank Accession no. NM_201277), CNN1 (r), 5'-GCCTCCTCCT GGTAGTAAGGG-3' (nt 1166-1146). IGF2(f), 5'-GACACCCTCCAGTTCGTCT GTG-3' (nt 667-688, GenBank Accession no. NM_000612), IGF2(r)，5'-GGTAGAGCAATCAGGGG ACGGTG-3' (nt 1043-1015). FGFR1(f), 5'-ATGGCA CCCGAGGCATTATT-3' (nt 2725-2744, GenBank Accession no. NM 000604), FGFR1(r), 5'-GGCTCAT GAGAGAAGACGGAAT-3' (nt 3125-3104). Reactions were prepared in duplicate and heated to $95^{\circ} \mathrm{C}$ for $10 \mathrm{~min}$ followed by 40 cycles of denaturation at $95^{\circ} \mathrm{C}$ for $10 \mathrm{~s}$, annealing at $55^{\circ} \mathrm{C}$ for $5 \mathrm{~s}$, and extension at $72^{\circ} \mathrm{C}$ for $20 \mathrm{~s}$. All PCR reactions were performed in duplicate. Standard curves (cycle threshold values vs template concentration) were prepared for each target gene and for the endogenous reference (human $\beta 2$-microglobulin) in each sample. The relative fold of differential expression was the ratio of the normalized value of each sample (AT/RT and Daoy medulloblastoma cell) to the normalized values of the controls (astrocyte SVG12 cell). To confirm the specificity of the PCR reaction, PCR products were electrophoresed in a $1.2 \%$ agrose gel.

\section{Immunohistochemistry}

Immunohistochemical staining was performed on AT/RT, DAOY medulloblastoma cell lines as well as $\mathrm{AT} / \mathrm{RT}$ and medulloblastoma tissue specimens. The $4 \mu \mathrm{m}$ paraffin sections of four AT/RT and six medulloblastoma were deparaffinized in xylene, rehydrated in a series of graded alcohols, and immunostained with antibodies against vimentin (ChemMate, DAKO, Glostrup, Denmark), epithelial membrane antigen (ChemMate, DAKO), neuronspecific enolase (ChemMate, DAKO), glial fibrillary acidic protein (ChemMate, DAKO), S-100 protein (ChemMate, DAKO), smooth muscle antigen (Chem-
Mate, DAKO), synatophysin (ChemMate, DAKO) and osteopontin (10A16; Immuno-Biological Laboratories, Gumma, Japan) Immunoreactive signals were detected with a mixture of biotinylated IgG antibody and peroxidase-conjugated streptavidin (LSAB2 system, DAKO).

\section{Results}

To elucidate the histogenesis and biology of AT/RT, we established an in vitro AT/RT cell line that was derived from a confirmed case of AT/RT (Figure 1a). The features of AT/RT-derived cells included the presence of large, pale, bland cells designated as 'rhabdoid' cells (Figure 1b). Rapidly dividing rhabdoid tumor cells dominated the culture (Figure 1b). The results of immunohistochemistry for this cell line were consistent with the diagnosis of AT/RT (ie, vimentin (VIM; Figure 1c), neuron-specific enolase (NSE; Figure 1d), smooth muscle actin (SMA; Figure 1e), cytokeratin (CK; Figure 1f), glial fibrillary acidic protein (GFAP; Figure 1g), epithelial membrane antigen (EMA; Figure 1h), and synatophysin (SYN; Figure 1i) were all positive). This strain of AT/RT cells could be passed stably for more than 25 passages without loss of growth, viability, or morphological and immunochemical features of the parental tumor, as indicated by the continued presence of the serial tumor markers that we identified in our previous study. ${ }^{3}$ To determine the cloning efficiency in soft agar, cells from the 25th passage were plated at $10^{3} \mathrm{cells} / \mathrm{ml}$. The plating efficiency was $58.4 \%$ in dish and $40.1 \%$ in soft agar after 2 weeks of culture (Figure 1j). Cell-doubling time of AT/RT cells was $21.3 \mathrm{~h}$, which was estimated from an in vitro growth curve of AT/RT. To further investigate the in vivo tumorigenic potential, $10^{6}$ of the AT/RT cells were injected into the right subcutaneous flank of SCID mice. After 2-4 weeks of implantation, the growth of solid tumor was observed. Histologically, infiltrations of compact rhabdoid-like, large round cells with vesicular nuclei and prominent nucleoli were found in these transplanted tumors (Figure 1k). Electron microscopy showed that the tumor cell had open chromatin and prominent nucleolus and perinuclear intermediate filaments (Figure 1l).

We next analyzed the chromosomal abnormality in this AT/RT cell line. The results of Giemsa banding revealed that these AT/RT cells were aneuploid. CGH was used to determine the copy number karyotype, and the results showed gains of chromosome regions 1p11p32, 1q21q32; 2p11p21, 2q11q21, 2q24q37; 3p21p26; 4q25; 5p14, 5q14, 5q23, 5q33q35; 6q15, 6q22; 7p11p22, 7q11q22; 8p12, 8p22, 8p23, 8q13q24; 10p11p13, 10q11q21; 11p12p15; 12p11, 12q11q24; 13q14q21, 13q31q32; 16q23; 20p12p13, 20q11q13; 21q11.2q22 and losses of chromosome regions $1 \mathrm{p} 36.3 ; 2 \mathrm{p} 24 \mathrm{p} 25$; 3p12; 6p23p25; 13q21q22, 13q32q34; 14q11q13, 

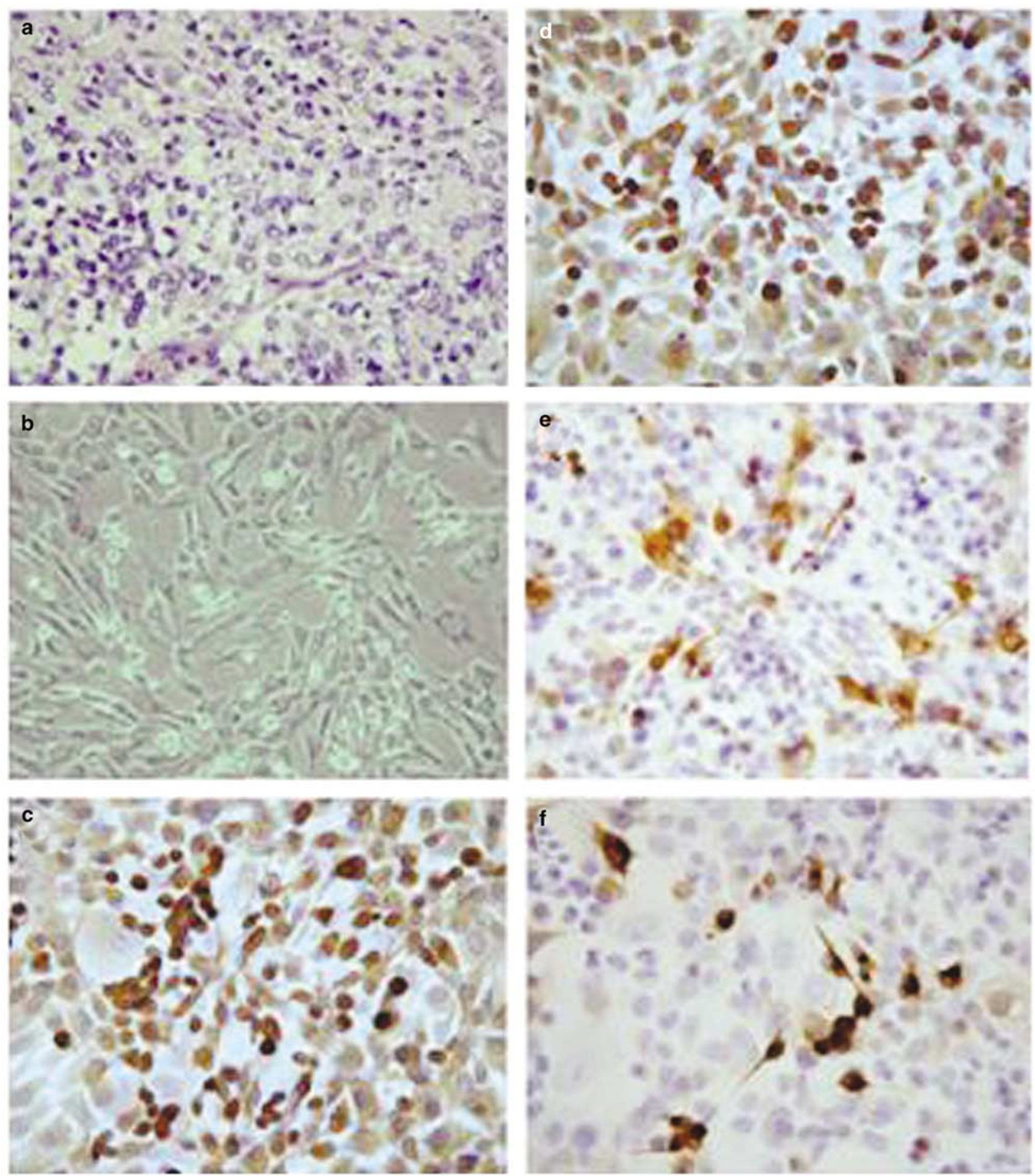

Figure 1 Establishment and characterization of an in vitro AT/RT-derived cell line. (a) Histological survey of a human AT/RT specimen. Magnification: $\times 200$. (b) In vitro cultivation for AT/RT-derived cell from the same patient. Magnification: $\times 400(\mathbf{c}-\mathbf{i})$ Immunohistochemical studies demonstrated positive immunoreactivity for VIM (C), NSE (D), SMA (E), CK (F), GFAP (G), EMA (H), and SYN (1I). Magnification: $\times$ 400. (j) AT/RT cells formed colonies in soft agar after 2 weeks of culture. Magnification: $\times 100$. (k) Histology of transplanted AT/RT cells in SCID mice. Magnification: $\times 100$. (l) Electron microscopy revealed tumor cells with prominent nuclei and bundles of intermediate filaments (bar: $2 \mu \mathrm{m}$ ).

14q22q32; 15q11q21; 18q11q23; 22q11.2q13 (Figure 2). These data are consistent with CGH results for the parental tumor.
Next, we analyzed the expression pattern of 7500 genes in AT/RT cells using cDNA microarray. DAOY medulloblastoma and astrocyte SVG12 cells were 

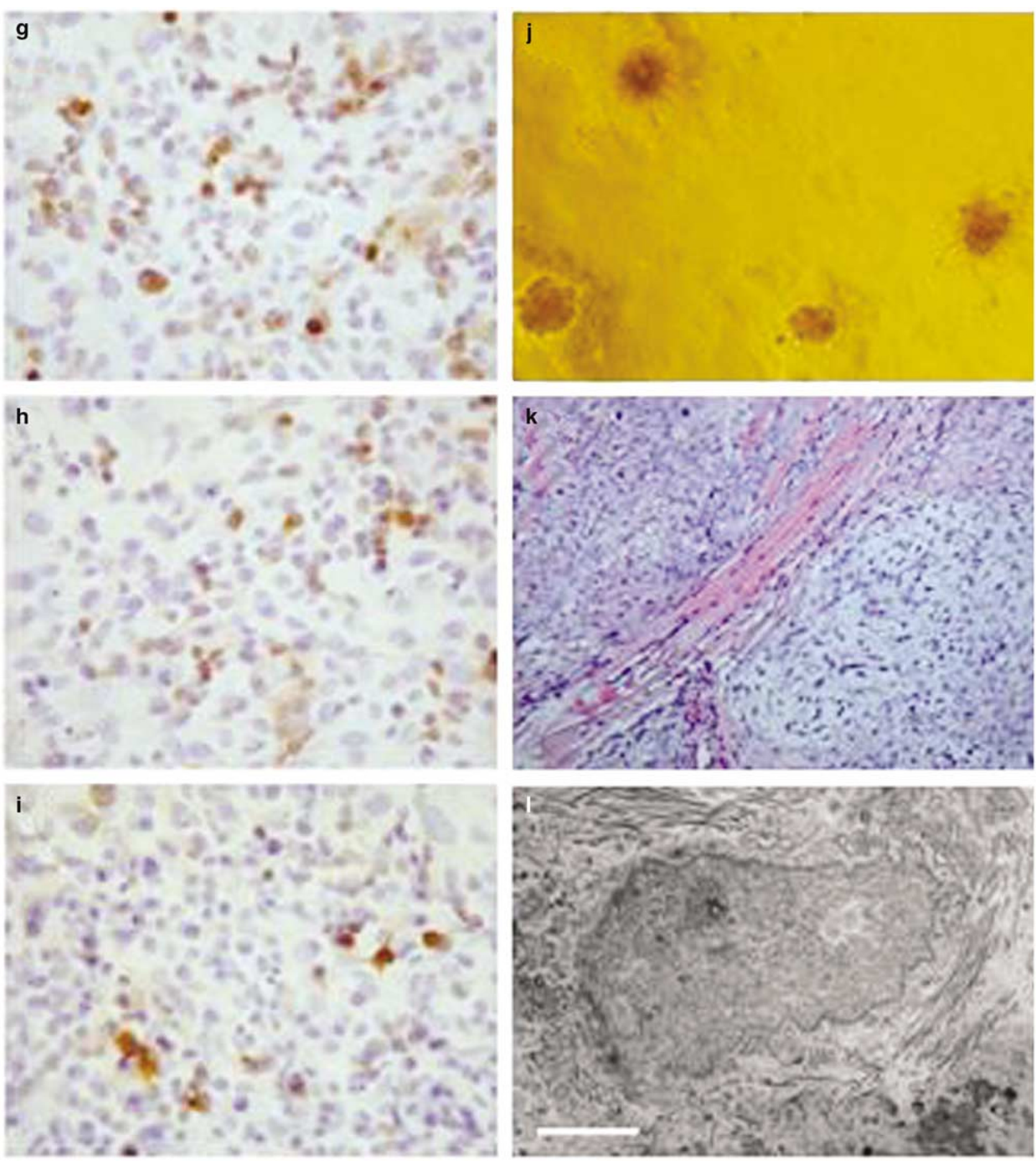

Figure 1 Continued.

compared to AT/RT cells. Experiments were repeated three times. On the basis of differences in the mean expression intensity values for each gene, a total of 114 genes significantly differed in their expression levels among astrocyte SVG-12, Daoy medulloblastoma and AT/RT cells by at least twofold ( $\geqq$ two-fold) in upregulation and 1/2-fold ( $\leqq 0.5$ fold) in downregulation $(P<0.01)$ when compiled with the hierarchical clustering method (Figure 3).
Of the 114 genes, the expression of 22 genes (FGFR1, DCN, ZNF3, PTS, LUM, COL1A2, CUL4B, CRABP2, MMP2, COL11A1, LMO4, CNN1, TNFSF7, SERINA3, DLK1, SPP1(OPN), PLA2R1, PDGFRA, HSPG2, TPM2, $M X 1$, and PCOLCE) was upregulated ( $\geqq$ fivefold) in AT/RT cells relative to astrocyte SVG12 cells, and 16 genes (CAV1, SPP1(OPN), MMP2, COL1A2, DCN, S100A10, LUM, SNCAIP, LMO4, COL6A1, ALDH2, ERG1, CAMK2G, CNN1, CDH11, 

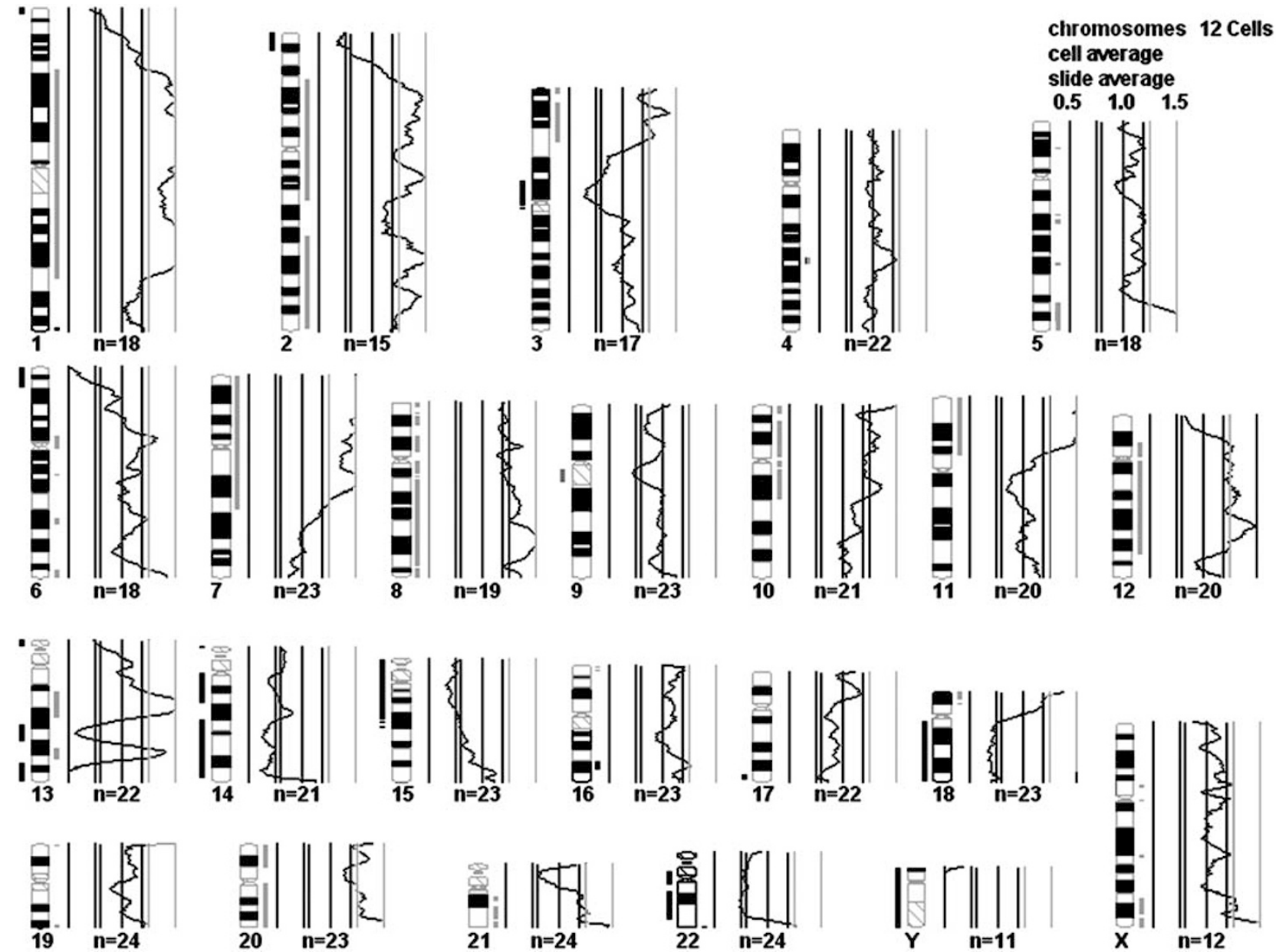

Figure 2 Ratio profile of chromosomal imbalance regions identified by CGH in AT/RT-derived cell line. The averaged ratio profile of CGH. The bar on the right of the chromosome idiogram indicates gain of chromosomal materials in AT/RT cells, and the bar to the left indicates loss of chromosomal materials in AT/RT cells.

and INA) was upregulated ( $\geqq$ five-fold) in AT/RT cells relative to DAOY medulloblastoma cells. The expression of 24 genes (FCGR2A, GSTA2, GPX1, PLAUR, LAMB1, GLRX, CDC42EP3, SGCD, SSB, PVALB, ZNF35, PKP2, BNC, ARHE, ENG, GNAI1, ADPRT, VCAM1, CCL7, TM4SF5, RASA1, CCNI, $P T P R C$, and THBD) in AT/RT cells was downregulated relative to both astrocyte and medulloblastoma cell lines (Figure 3).

To further validate the microarray analysis findings of increased AT/RT-related genes expression, we performed real-time RT-PCR analyses to examine the mRNA level of eight target genes including OPN, metalloprotease-2 (MMP-2), lumican (LUM), decorin $(D C N)$, platelet-derived growth factor receptor $\alpha$ (PDGFRA), caplonin 1 (CNN1), insulin growth factor
2 (IGF2), and fibroblast growth factor receptor 1 (FGFR1) in astrocyte SVG12 cells, DAOY medulloblastoma cells, and AT/RT cells (Figure 4). In agreement with our microarray analysis findings, AT/RT cells, but not the other two cells, showed significantly higher expression levels of all genes tested except for IGF2 (Figure 4). The expression ratio of lumican was highest in both AT/RT and DAOY medulloblastoma cells (37.1 and 24.9 , respectively). Of note, the only $O P N$ ratio less than 1.0 was found in DAOY medulloblastoma cells. The AT/RT OPN expression level was more than 32-fold higher than the expression in medulloblastoma cells (8.8/0.27), which was the most profound difference among the eight target genes (Figure 4).

Figure 3 Differential expression of genes in AT/RT cell vs astrocyte SVG12 cell and AT/RT cell vs DAOY medulloblastoma cell. Hierarchical clustering analysis of 114 genes selected from 7500 genes set, showing upregulated ( $\geqq$ two-fold; red color) or downregulated ( $\leqq 0.5$-fold; green color) expression profiles in AT/RT cell compared with astrocyte SVG12 cell and DAOY medulloblastoma cell. The data are represented as log ratio of the mean calculated from three replicates expression values provided by GeneSpring and J-Express software. 


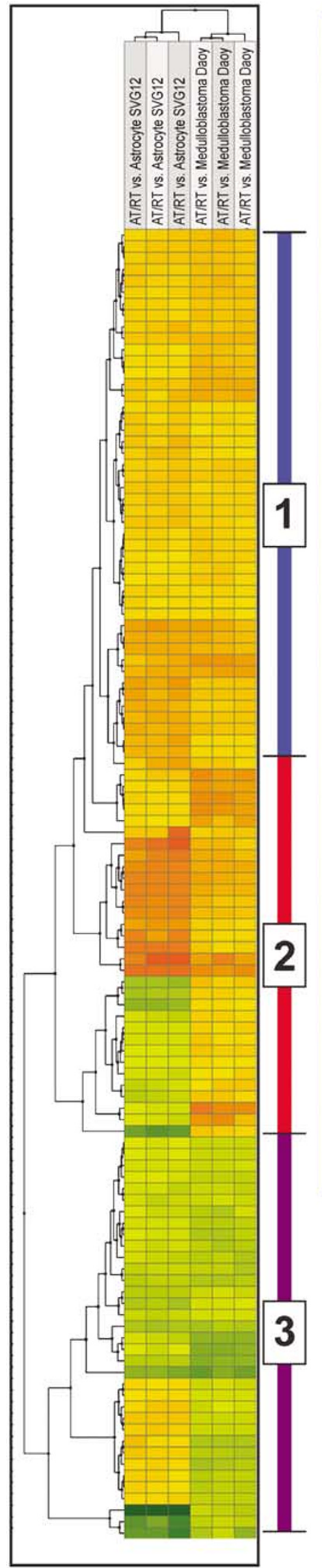

\begin{tabular}{|c|c|}
\hline Accession & Symbol \\
\hline AA598611 & NR4A2 \\
\hline AA489743 & IFIT1 \\
\hline AA487893 & TM4SF1 \\
\hline AA464197 & MSX1 \\
\hline AA701929 & BYSL \\
\hline AA778663 & TNFSF9 \\
\hline N64741 & SNAI2 \\
\hline A1261660 & EPHB4 \\
\hline AA410437 & B3GALT6 \\
\hline N74882 & DLX5 \\
\hline AA487557 & EPLIN \\
\hline H41122 & GABRD \\
\hline R55075 & MPV17 \\
\hline AA410381 & SNCAIP \\
\hline AA136983 & CDH11 \\
\hline AA676471 & EIF3S9 \\
\hline AA455882 & PPP1R3F \\
\hline AA447661 & PA26 \\
\hline AA455210 & PDGFRL \\
\hline AA446884 & RFP2 \\
\hline AA485743 & FLJ14675 \\
\hline N94468 & JUNB \\
\hline AA461456 & COL5A2 \\
\hline R71440 & SERPINH2 \\
\hline N66737 & COL2A1 \\
\hline AA779457 & BMP5 \\
\hline AA436260 & ATP9A \\
\hline W73810 & EMP3 \\
\hline R11526 & PTMS \\
\hline W87611 & NFIB \\
\hline H39192 & MAPK7 \\
\hline AA400234 & MEN1 \\
\hline AA150538 & RIPK1 \\
\hline AA234897 & MEF2C \\
\hline AA670200 & PCOLCE \\
\hline AA427561 & HSPG2 \\
\hline R54846 & FGFR1 \\
\hline AA448015 & INA \\
\hline H27986 & LMO4 \\
\hline AA477400 & TPM2 \\
\hline AA088564 & ZNF3 \\
\hline AA448478 & G1P3 \\
\hline AA406020 & G1P2 \\
\hline H23235 & PDGFRA \\
\hline AA399519 & CNN1 \\
\hline AA780712 & CULAB \\
\hline
\end{tabular}

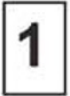

\begin{tabular}{|l|l|}
\hline AA873564 & TLE2 \\
\hline AA056601 & CAMK2G \\
\hline AA486628 & EGR1 \\
\hline AA444051 & S100A10 \\
\hline H99676 & COL6A1 \\
\hline AIS90849 & ALDH2 \\
\hline A28153 & MMP2 \\
\hline AA456886 & MX1 \\
\hline AA701996 & DLK1 \\
\hline AA490172 & COL1A2 \\
\hline AA453712 & LUM \\
\hline AA419251 & PTS \\
\hline A1347622 & TNFSF7 \\
\hline AA598508 & CRABP2 \\
\hline AA086038 & PLA2R1 \\
\hline R31701 & COL11A1 \\
\hline AA704242 & SERPINA3 \\
\hline AA775616 & SPP1 \\
\hline H64138 & DCN \\
\hline AA488676 & BASP1 \\
\hline AA137031 & SOCS2 \\
\hline AAS77166 & MYL9 \\
\hline T60048 & ACTG2 \\
\hline AA868008 & H4F \\
\hline AA001746 & DC2 \\
\hline AA187351 & RRM2 \\
\hline AA995282 & FHL2 \\
\hline T53298 & IGFBP7 \\
\hline H06571 & A2M \\
\hline W96134 & JUN \\
\hline AA055835 & CAV1 \\
\hline R13558 & ALCAM \\
\hline W46900 & CXCL1 \\
\hline & \\
\hline
\end{tabular}

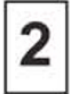

\begin{tabular}{|l|l|}
\hline AA634109 & FCGR2A \\
\hline H29484 & SSB \\
\hline N64607 & ZNF35 \\
\hline H66158 & PKP2 \\
\hline H74265 & PTPRC \\
\hline H59861 & THBD \\
\hline AA010609 & PVALB \\
\hline AA040170 & CCL7 \\
\hline W84524 & TM4SF5 \\
\hline N99098 & RASA1 \\
\hline R26526 & BNC \\
\hline AA234982 & SGCD \\
\hline AA406420 & GNAI1 \\
\hline AA485362 & GPX1 \\
\hline AA213816 & CDC42EP3 \\
\hline AA189050 & ADPRT \\
\hline T73468 & GSTA2 \\
\hline AA446251 & LAMB1 \\
\hline N58511 & CCNI \\
\hline AA446108 & ENG \\
\hline AA291163 & GLRX \\
\hline AA664056 & PREP \\
\hline N54596 & IGF2 \\
\hline H17047 & ZNF133 \\
\hline H09461 & 13CDNA73 \\
\hline R45941 & PTPRN \\
\hline AA485214 & NUCB2 \\
\hline R93124 & AKR1C-pseudo \\
\hline AW073355 & HPCAL1 \\
\hline T71991 & CREG \\
\hline AA625859 & NAP4 \\
\hline W25035 & FKBP8 \\
\hline AA455222 & PLAUR \\
\hline AA443302 & ARHE \\
\hline H16637 & VCAM1 \\
\hline & \\
\hline
\end{tabular}

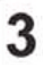

Cluster method: Average Linkage (UPGMA)

Distance metric: Euclidean

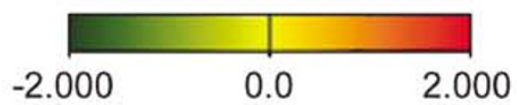


To investigate the expression of OPN in clinical samples of AT/RT patients, immunohistochemistry assays were performed. Consistent with the results

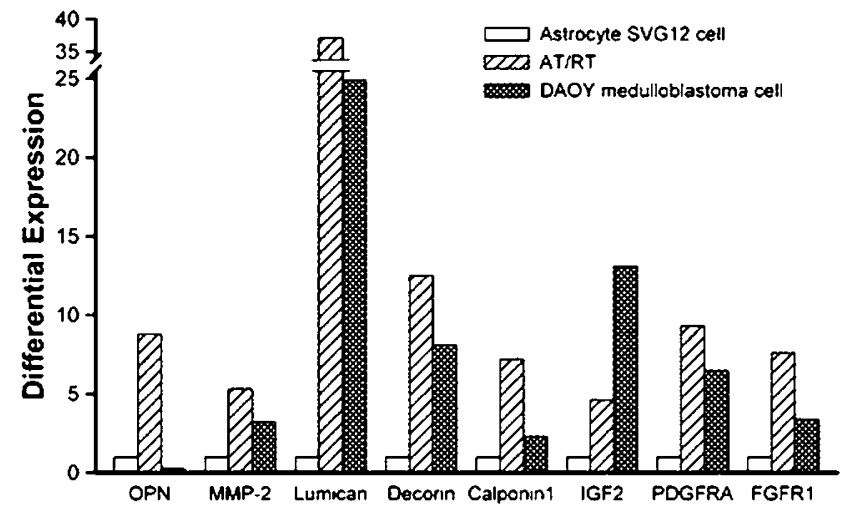

Figure 4 Relative quantitation of gene expression levels in AT/RT cell, astrocyte SVG12 cell and DAOY medulloblastoma cell by real-time RT-PCR. Comparison of the gene expressions of OPN (SPP1), MMP2, LUM, DCN, PDGFRA, CNN1, IGF2, and FGFR1. The relative ratio of differential expression in AT/RT and DAOY medulloblastoma cell was normalized to the ratio of astrocyte SVG12 cell.
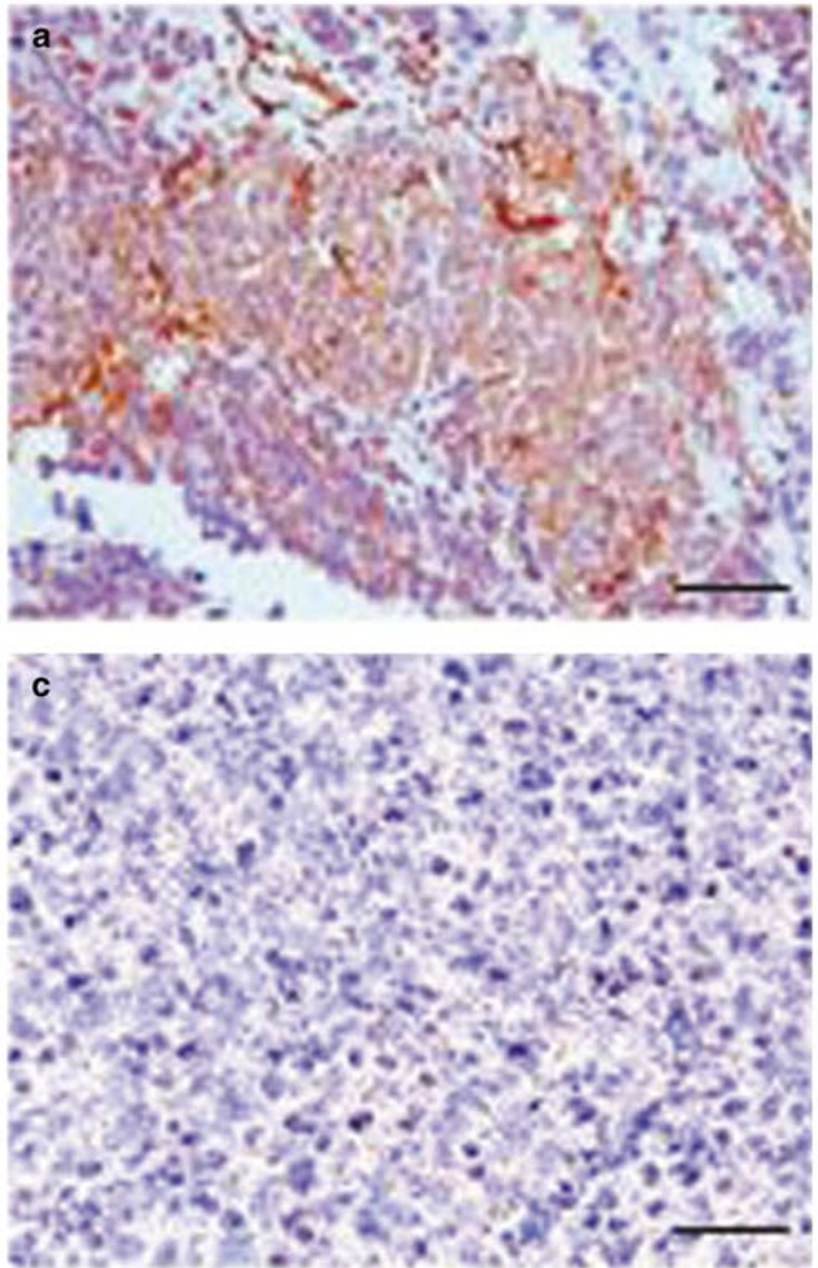

of in vitro AT/RT cells, we observed increased expressions of OPN protein in four AT/RT patients (Figure 5a). The immunohistochemistry survey also confirmed that the expression of OPN was strongly enhanced in tumor lesions and especially in rhabdoid-type cells of AT/RT specimens (Figure 5b). In contrast, only weakly positive signals were detected in six medulloblastoma patients (Figure 5c and d).

\section{Discussion}

In the present study, we successfully established an ATRT-derived cell line that could be passaged for more than 25 generations without loss of growth, viability, or AT/RT markers typical of its parental tumor counterpart. Further evidence for the malignant nature of this AT/RT-derived cell line is its rapid doubling time, high cloning efficiency in soft agar (Figure 1j), and ability to form solid tumors in SCID mice (Figure 1k). Chromosome analysis of the AT/RT cell line showed deletion of 22.q11, consistent with previous cytogenetic reports of AT/RT.,
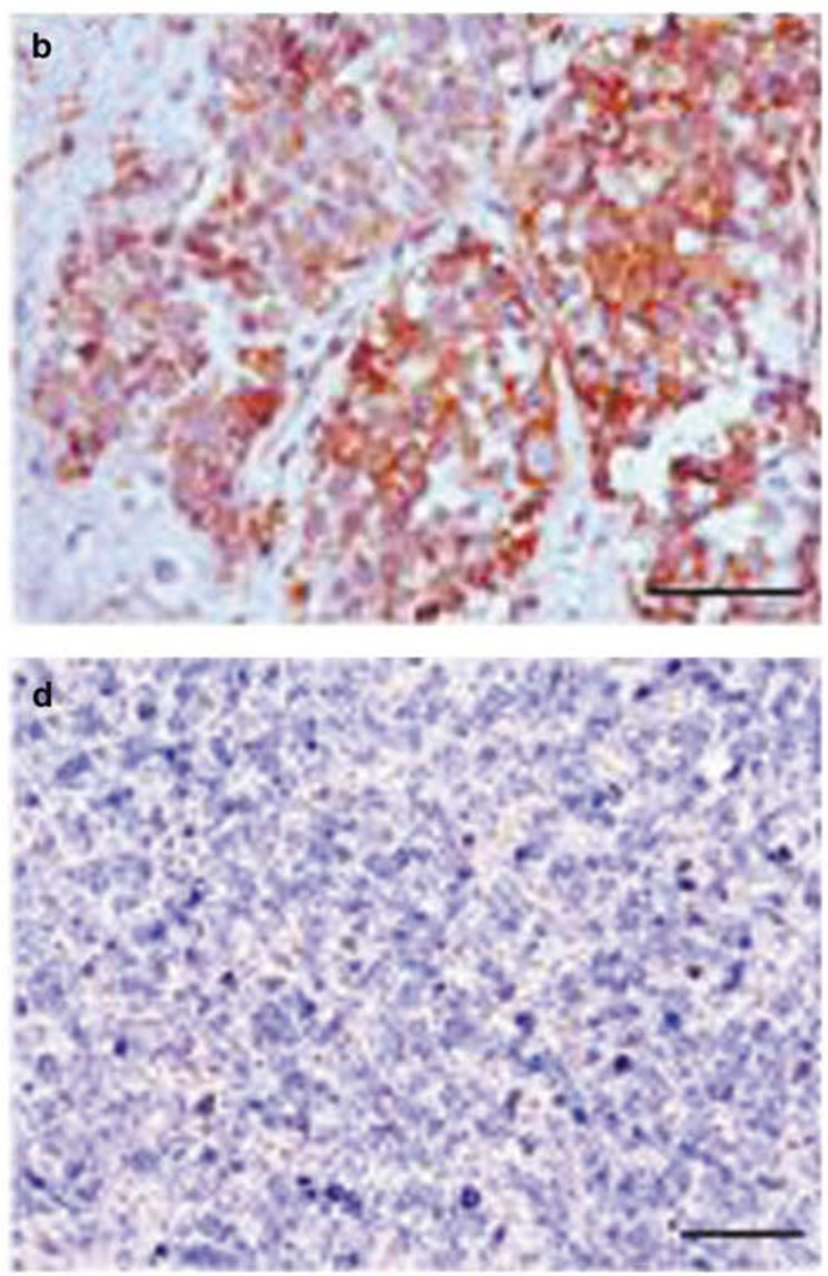

Figure 5 Immunolocalization of OPN in AT/RT tissues. (a,b) The increased expressions of OPN protein in AT/RT patients (a) and strong enhancement in the tumor cells (b). Weakly positive signals were seen in medulloblastoma tissues (c, d). Magnification: $\times 400$. 
Chromosome regions 3p12, 6p23p25, 13q21q22, 13q32q34, 14q11q13, 14q22q32, 15q11q21, and 8q11q23 were also found deleted (Figure 2). Microarray assays further revealed that the OPN gene was upregulated by at least five-fold in AT/RT cells relative to both astrocyte SVG12 and DAOY medulloblastoma cells (Figure 3). The results of real-time RT-PCR also demonstrated that the expression level of the $O P N$ gene was significantly higher in AT/RT cells than in astrocyte SVG12 (nine-fold) and DAOY medulloblastoma (32-fold) cells (Figure 4). Overexpression of OPN was further confirmed in the pathological sections of AT/RT patients as compared with medulloblastoma specimens.

OPN, a bone matrix glycoprotein, is thought to function as a modulator for bone resorption and remodeling. ${ }^{14}$ The mechanisms of $\mathrm{OPN}$ in the progression of malignancy may possibly involve binding of integrin via integrin-mediated signaling or inducing the activities of metalloproteinase (MMP family) members, ${ }^{14,15}$ both of which are believed to be responsible for adhesion and migration of cancers. Recent clinical reports have demonstrated that OPN is associated with decreased survival times in several metastatic neoplasms including breast cancer, prostate cancer, gastric cancer, colon cancer, head neck squamous carcinoma, hepatocellular carcinoma, and ovary cancer. ${ }^{16,17}$ Our data showed that the expression of OPN RNA and protein was high in AT/RT cells and clinical tissues of AT/RT patients, but not in patients with medulloblastoma. Previous studies have pointed out that the expression level of OPN differed significantly between individual astocytoma tissues and appeared to correlate with their malignancy grade and invasive potential. ${ }^{18}$ In a recent study, osteopontin was also found to promote the attachment of malignant astrocytoma cells to become more metastasized and invasive. ${ }^{19} \mathrm{On}$ the basis of our in vitro and in vivo observations, the high expression of OPN in AT/RT may be associated with tumor cell invasion and dissemination, which may lead to the unique clinical character of the disease and poor outcome of AT/RT patients.

In addition to the $O P N$ gene, PDGFRA, FGFR1, and $M M P-2$ genes were found differentially expressed among AT/RT, astrocyte SVG12, and DAOY medulloblastoma cells. We noted that the expression level of PDGFRA was higher in AT/RT cells than in the astrocyte and medulloblastoma cell lines (Figures 3 and 4). Recent studies showed that overexpressed PDGFRA and the RAS/MAPK signaling pathways played important roles in medulloblastoma metastasis. ${ }^{20}$ FGFR1 signaling was important for the earliest stage of tumor establishment, and FGFR1 promoted in vivo tumor proliferation and activated extracellular signal-regulated kinase through transcriptionally upregulated $O P N .^{21}$ A recent study also revealed that OPN stimulated tumor growth and activation of proMMP2 through NF $\kappa \mathrm{B}$-mediated induction of mem- brane type $1 \mathrm{MMP}$ in melanoma. ${ }^{22}$ In our study, the results of real-time RT-PCR confirmed that the genes for PDGFRA, MMP2, and FGFR1 were highly expressed in AT/RT (Figures 3 and 4), implying a role of the corresponding proteins in tumor metastasis. The upexpression of several other genes was also shown in AT/RT cells by cDNA microarray analysis (Figure 3). These include tumor proliferation and cancer-related genes EPHB4, COL5A2, COI2A1, PA26, JUNB, PTS, EMP3, ERG1, MAPK7, $P L A 2 R 1$, HSPG2, DCN, IGF2, and ALCAM, as well as the myogenesis gene markers-calponin 1 (CNN1), tropomyosin 2 (TPM2), myosin light polypeptide 9 (MYL9), and actin gamma 2 (ACTG2). ${ }^{23-27}$ These findings are compatible with the pathological features of our AT/RT cells in which the marker of smooth muscle actin (SMA) showed positive immunoreactivity (Figure 1h). Furthermore, the expression of $Z N F 35$, THRB, and RASA1 genes was significantly lower in AT/RT cells than in astrocyte and medulloblastoma cell lines (Figure 3). These genes are of particular interest because the ZNF35 and $T H R B$ genes, functioning as tumor suppressor genes, were found mutated in cervical, lung, breast and renal cell cancers. ${ }^{28}$ RASA1, a positional candidate gene with a mutation for capillary malformation-arteriovenous malformation, ${ }^{29}$ might be involved in the aggressive behavior of AT/RT tumor. Thus, these downregulated genes should be scrutinized in future studies of AT/RT pathogenesis.

In sum, this small-scaled study has demonstrated the overexpression of OPN in AT/RT in comparison to both in vitro and in vivo medulloblastoma. Known medulloblastoma cell lines show some variations in their biological characteristics, and the present study only compared AT/RT cells with DAOY medulloblastoma cell line. To evaluate the significance of OPN in AT/RT, a larger number of AT/RT and medulloblastoma tissue immunostaining is required. However, despite the small sample size, this investigation suggests the potential value of OPN as a biomarker for diagnosis of AT/RT. In the future, a larger number of tumor cases can be incorporated to provide more conclusive evidence.

\section{Acknowledgements}

This study was supported by grants from Taipei Veterans General Hospital (93-B189), the Joint Projects of VTY (92-P1-07/08) and UTVGH (93-P104/06), Yen-Tjing Ling Medical Foundation, and National Science Council (NSC-92 and 93). We thank the National Microarray and Gene Expression Analysis Core Facility of National Yang-Ming University for the excellent technique support. We also thank Professor Shang-Ming Yu (National Yang-Ming University) for electron microscopy study and Dr Li-Jung Juan (National Health Research Institutes of Taiwan) for comments on the manuscript. 


\section{References}

1 Rorke LB, Packer RJ, Biegel JA. Central nervous system atypical teratoid/rhabdoid tumors of infancy and childhood: definition of an entity. J Neurosurg 1996; 85:56-65.

2 Burger PC, Yu IT, Tihan T, et al. Atypical teratoid/ rhabdoid tumor of the central nervous system: a highly malignant tumor of infancy and childhood frequently mistaken for medulloblastoma. Am J Surg Pathol 1998;22:1083-1092.

3 Ho DMT, Hsu CY, Wong TT, et al. Atypical teratoid/ rhabdoid tumor of the central nervous system: a comparative study with primitive neuroectodermal tumor/medulloblastoma. Acta Neuropathol 2000;99: 482-488.

4 Oka H, Scheithauer BW. Clinicopathological characteristics of atypical teratoid/rhabdoid tumor. Neurol Med Chir (Tokyo) 1999;39:510-518.

5 Emadian SM, McDonald JD, Gerken SC, et al. Correlation of chromosome 17p loss with clinical outcome in medulloblastoma. Clin Cancer Res 1996;2:1559-1564.

6 Tong CYK, Hui ABY, Yin X-L, et al. Detection of oncogene amplifications in medulloblastomas by comparative genomic hybridization and array-based comparative genomic hybridization. J Neurosurg (Pediatrics 2) 2004;100:187-193.

7 Biegel JA, Burk CD, Parmiter AN, et al. Molecular analysis of a partial deletion of $22 q$ in a central nervous system rhabdoid tumor. Gene Chromosomes Cancer 1992;5:104-108.

8 Biegel JA, Allen CS, Kawasaki K, et al. Narrowing the critical region for a rhabdoid tumor locus in $22 \mathrm{q} 11$. Gene Chromosomes Cancer 1996;16:94-105.

9 Versteege I, Sevenet N, Lange J, et al. Truncating mutations of hSNF5/INI1 in aggressive pediatric cancer. Nature 1998;394:203-206.

10 Lopez-Gines C, Cerda-Nicolas M, Kepes J, et al. Complex rearrangement of chromosomes 6 and 11 as the sole anomaly in atypical teratoid/rhabdoid tumors of the central nervous system. Cancer Genet Cytogen 2000;122:149-152.

11 Sawyer JR, Goosen LS, Swanson CM, et al. A new reciprocal translocation $(12 ; 22)(\mathrm{q} 24.3 ; \mathrm{q} 11.2-12)$ in a malignant rhabdoid tumor of the brain. Cancer Genet Cytogen 1998;101:62-67.

12 Chen YJ, Chen PJ, Lee MC, et al. Chromosomal analysis of hepatic adenoma and focal nodular hyperplasia by comparative genomic hybridization. Gene Chromosomes Cancer 2002;35:138-143.

$13 \mathrm{Xu}$ Q, Dziejman M, Mekalanos JJ. Determination of the transcriptome of Vibrio cholerae during intraintestinal growth and midexponential phase in vitro. Proc Natl Acad Sci USA 2003;100:1286-1291.

14 Furger KA, Menon RK, Tuck AB, et al. The functional and clinical roles of osteopontin in cancer and metastasis. Curr Mol Med 2001;1:621-632.

15 Hirama M, Takahashi F, Takahashi K, et al. Osteopontin overproduced by tumor cells acts as a potent angiogenic factor contributing to tumor growth. Cancer Lett 2003;198:107-117.

16 Coppola D, Szabo M, Boulware D, et al. Correlation of osteopontin protein expression and pathological stage across a wide variety of tumor histologies. Clin Cancer Res 2004;10:184-190.

$17 \mathrm{Kim} \mathrm{JH}$, Skates SJ, Uede T, et al. Osteopontin as a potential diagnostic biomarker for ovarian cancer. JAMA 2002;287:1671-1679.

18 Saitoh Y, Kuratsu JI, Takeshima H, et al. Expression of osteopontin in human glioma. Lab Invest 1995;72:55-63.

19 Ding Q, Stewart Jr J, Prince CW, et al. Promotion of malignant astrocytoma cell migration by osteopontin expressed in the normal brain: differences in integrin signaling during cell adhesion to osteopontin versus vitronectin. Cancer Res 2002;62:5336-5343.

20 MacDonald TJ, Brown KM, Lafleur B, et al. Expression profiling of medulloblastoma: PDGFRA and RAS/ MAPK pathway as therapeutic targets for metastatic disease. Nat Genet 2001;29:143-152.

21 Freeman KW, Gangula RD, Welm BE, et al. Conditional activation of fibroblast growth factor (FGFR) 1, but not FGFR2, in prostate cancer cells leads to increased osteopontin induction, extracellular signal-regulated kinase activation, and in vivo proliferation. Cancer Res 2003;63:6237-6243.

22 Philip S, Bulbule A, Kundu GC. Osteopontin stimulates tumor growth and activation of promatrix metalloproteinase-2 through nuclear factor-kBmediated induction of membrane type 1 matrix metalloproteinase in murine melanoma cells. J Biol Chem 2001;276:44926-44935.

23 Mackay A, Jones C, Dexter T, et al. cDNA microarray analysis of genes associated with ERBB2 (HER2/neu) overexpression in human mammary luminal epithelial cells. Oncogene 2003;22:2680-2688.

24 Cromer A, Carles A, Millon R, et al. Identification of gene associated with tumorigenesis and metastatic potential of hypopharyngeal cancer by microarray analysis. Oncogene 2004;23:2484-2498.

25 Fischer H, Stenling R, Rubio C, Lindblom A. Colorectal carcinogenesis is associated with stromal expression of COL11A1 and COL5A2. Carcinogenesis 2001;22: 875-878.

26 van Dartel M, Cornelissen PW, Redeker S, et al. Amplification of $17 \mathrm{p} 11.2$ approximately p12, including PMP22, TOP3A, and MAPK7, in high-grade osteosarcoma. Cancer Genet Cytogen 2002;139:91-96.

27 Banerjee AG, Bhattacharyya I, Lydiatt WM, et al. Aberrant expression and localization of decorin in human oral dysplasia and squamous cell carcinoma. Cancer Res 2003;63:7769-7776.

28 Kohno T, Takayama H, Hamaguchi M, et al. Deletion mapping of chromosome $3 p$ in human uterine cervical cancer. Oncogene 1993;8:1825-1832.

29 Eerola I, Boon LM, Mulliken JB, et al. Capillary malformation-arteriovenous malformation, a new clinical and genetic disorder caused by RASA1 mutations. Am J Hum Genet 2003;73:1240-1249. 